\title{
Disequilibrium dynamics in a Keynesian model with time delays
}

\author{
Luca Gori ${ }^{*}$ Luca Guerrini ${ }^{\dagger} \bullet$ Mauro Sodini ${ }^{\ddagger}$
}

May 19, 2017

\begin{abstract}
The aim of this research is to analyse a Keynesian goods market closed economy by considering a continuous-time setup with fixed delays. The work compares dynamic results based on linear and nonlinear adjustment mechanisms through which the aggregate supply (production) reacts to a disequilibrium in the goods market and consumption depends on income at a preceding date. Both analytical and geometrical (stability switching curves) techniques are used to characterise the stability properties of the stationary equilibrium.
\end{abstract}

Keywords Delay differential equations; Keynesian model; Stability crossing curves

JEL Codes C62; E12; E32; E62

AMS Codes $34 \mathrm{~K} 18 ; 34 \mathrm{~K} 20$

\section{Introduction}

Macroeconomic models of Keynesian tradition have always played an influential role in the economic literature and, amongst other things, they have represented a natural starting point for the formulation of economic dynamic problems (e.g., Allen, 1959). In this respect, there are at least two distinct modelling approaches. On the one hand, there is plenty of research dealing with equilibrium dynamics, i.e. models in which the equality between aggregate demand and aggregate supply (production) in the real or goods market holds at every date. On the other hand, several works also account for disequilibrium dynamics, i.e. models in which economic dynamics is studied based on the assumption that aggregate demand can be different from aggregate production. Within this vast stream of research several issues were addressed: for instance, the study of problems related to international trade or those accounting for the nonneutrality of money (e.g., Chiarella and Flaschel, 2000). In addition, some Keynesian intuitions have also led to the building on models unusual in the tradition of economic literature due to their non-trivial mathematical properties. To this purpose, we mention here the works of 1) Asada and Yoshida (2001), the aim of which is to study the macroeconomic effects of a "policy lag" (Asada and Yoshida, 2001, p. 282) in a nonlinear dynamic version of a Keynesian model. In

${ }^{*}$ L. Gori (corresponding author), Department of Political Science, University of Genoa, Piazzale E. Brignole, 3a, I-16125 Genoa (GE), Italy, e-mail: luca.gori@unige.it or dr.luca.gori@gmail.com, tel.: +39010 2099503 , fax: +390102095536 .

${ }^{\dagger}$ L. Guerrini, Department of Management, Polytechnic University of Marche, Piazza Martelli 8, 60121, Ancona (AN), Italy, e-mail: luca.guerrini@univpm.it, tel.: +390712207055.

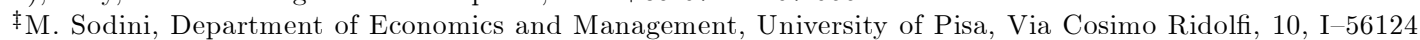
Pisa (PI), Italy, e-mail: mauro.sodini@unipi.it, tel.: +390502216 234, fax: +390502210603. 
particular, the authors characterise the dynamic properties of the resulting system governed by delay differential equations finding that too long a delay in implementing the policy may make the stabilising effect of the public expenditure ineffective; 2) Cánovas and Ruiz Marín (2006), who develop a non-autonomous model in discrete time finding some analytical and simulative results on the existence of chaotic dynamics that are well suited to describe business cycle fluctuations; 3) Böhm (2006), who modifies the standard multiplier-accelerator model by adding several kinds of random components acting either on the multiplier or the accelerator or, alternatively, on both of them.

It is interesting to note that part of the (dynamic) Keynesian literature has been developed in discrete time (usually equilibrium models) and another part has instead built on in continuous time (usually based on disequilibrium models in which dynamic adjustment mechanisms depend on the excess demand in the goods market). ${ }^{1}$ Without the aim of deepening in a comprehensive and thoughtful way this issue here, it is remarkable however that some textbooks on economic dynamics (Shone, 2001; Gandolfo, 2010) introduce the equilibrium Keynesian paradigm and its disequilibrium counterpart as if they were one the other one's interface depending on the mathematical setup used (i.e., discrete time or continuous time). ${ }^{2}$ Nonetheless, it is important to emphasise that equilibrium and disequilibrium dynamics have different meanings regardless of whether the model is built on in discrete time or continuous time. Actually, in a dynamic environment in which the aggregate demand is always equal to the aggregate supply all the effects related to the working of the principle of effective demand of Keynesian tradition are sterilised. According to this principle, aggregate demand determines the level of both actual production and employment and then the aggregate supply reacts to a disequilibrium in the goods market depending on the sign of the excess demand. This principle defines a disequilibrium paradigm with respect to which the equilibrium is a possible event that cannot however be imposed as an assumption.

Compared to the Keynesian tradition that in the recent past has used discrete maps to analyse equilibrium models, there is a burgeoning (more recent) interest in the analysis of discrete-time disequilibrium Keynesian models (where the dynamics is characterised by difference equations) focusing on the study of the interactions between real markets and financial markets (Westerhoff, 2012; Naimzada and Pireddu, 2014a, 2014b, 2015). Irrespective of the specific content of each single work, however, it is relevant to highlight that this literature has introduced nonlinear adjustment mechanisms with respect to which the goods market reacts depending on the sign of the excess demand. This makes possible observing models with an unstable market equilibrium where there exists a chaotic attractor able to capture the dynamics of the system (non-explosive dynamics). Indeed, the assumption of nonlinear adjustment mechanisms may help to capture and explain in a better way the (business) cycles observed in aggregate variables (income and employment) than a model with a linear adjustment mechanism in the goods market (where instability is a synonym for explosive dynamics). Business cycle, in fact, is a typical (empirical) feature of several developed economies and its (theoretical) explanation should not be based only on rational expectations frameworks with external or random perturbations (DSGE Keynesian models $\left.^{3}\right)$. This is because fluctuations are often an intrinsic phenomenon of market behaviours, so that models able to explain them endogenously are highly valuable.

\footnotetext{
${ }^{1}$ See Chiarella et al. (2000) for a survey of this issue.

${ }^{2}$ For a discussion on continuous-time and discrete-time versions of Keynesian macroeconomic models see Asada et al. (2010).

${ }^{3}$ See, for instance, the works of Smets and Wouters (2003) and Christiano et al. (2005). Although this approach is still the dominant paradigm in the literature, it should honestly be mentioned that it has been seriously questioned by some influential scholars (Mankiw, 2001; Solow, 2004). This is essentially because of the working of the rational expectations hypothesis in forward-looking economic models and its failure to adequately capture some empirical facts, as is pointed out by Estrella and Fuhrer (2002).
} 
A peculiar characteristic of this kind of disequilibrium discrete-time models is that the adjustment mechanism of aggregate consumption has the same timing as the adjustment mechanism in the goods market. This assumption makes the analysis of the dynamic model easier but it contributes to let the theoretical framework less able to describe the functioning of actual markets. The purpose of the present article is to generalise Naimzada and Pireddu (2014a) by considering a more flexible dynamic environment characterised by differential equations with two fixed delays. ${ }^{4}$ In particular, the work analyses four different formulations of adjustment mechanisms within a disequilibrium Keynesian goods market model. One of this mechanism generalises the classical linear adjustment process described by Ferguson and Lim (2003) in a discrete-time setup, according to which the change in income is a linear function of the disequilibrium in the goods market. Amongst other things, this exercise shows a peculiar characteristic of this kind of models, i.e. when aggregate production reacts quickly to a positive or negative excess demand in the goods market, the economic system is stable regardless of the timing with which (current) aggregate consumption reacts to aggregate production (corridor of stability). Another important point, however, is that despite linear delay differential equations systems can generate Hopf bifurcations, in this case this phenomenon has little interest as cyclical trajectories tend to diverge for several parameter values (this result can also be verified by applying the analytical techniques based on the central manifold theory). This implies that although the model is not able to explain persistent fluctuations, it is able however to show the existence of fluctuations (business cycles) that dampen more slowly in comparison with those generated by its discretetime counterpart. As a second exercise, this work modifies the previous adjustment mechanism by considering that the rate of change in income $\dot{Y}(t) / Y(t)$ (instead of $\dot{Y}(t)$ ) is a linear function of the disequilibrium in the goods market. In this case, the model is able to generate persistent fluctuations but it still continues to have some modelling shortcomings, that is for large values of the time delays or for initial conditions far enough from the stationary equilibrium it may produce divergent trajectories. In order to overcome this concern, the article considers two models reproducing in a delay differential equations context the nonlinear adjustment mechanism introduced by Naimzada and Pireddu (2014a) in a discrete-time setup, showing that this mechanism is well suited to describing business cycle dynamics in a more general context than those used in the existing literature.

From a mathematical point of view, all these models qualitatively share the same linear approximation. Therefore, the local stability properties and some results of global stability can safely be used in a unified context by adopting analytical findings (Piotrowska, 2007) and the stability crossing curves technique developed by Gu et al. (2005). This similarity, however, is lost when one considers dynamics generated by parameter configurations far enough from bifurcation values. This is pointed out in this study by using numerical simulations.

The rest of the article proceeds as follows. Section 2 sets up a static Keynesian model and then turns to consider a continuous-time dynamic framework with fixed delays. Then, it introduces different specifications of adjustment mechanisms to capture disequilibrium reactions in the goods market. By using analytical and geometrical (stability switching curves) techniques, Sections 3, 4 and 5 characterise local stability properties and local bifurcations of equilibria of the resulting one-dimensional delay differential equation system. In particular, Section 3 is devoted to the study of stability properties of the market equilibrium when there exists a double delay: one delay is related to the dependence of current consumption on the aggregate income (production) prevailing in a previous period; the other delay is due to the existence of an adjustment mechanism with which the economy reacts to an excess demand in the goods market. Section 4 analyses the case in which current consumption depends on current production (instead

\footnotetext{
${ }^{4}$ See, for instance, Matsumoto and Szidarovsky (2016) for an analysis of an economic model with a mathematical structure similar to ours.
} 
of delayed production). Sections 3 and 4 also provide some analytical results of global stability. Section 6 presents some numerical simulations and Section 7 outlines the conclusions.

\section{The model}

We consider the functioning of a Keynesian (goods market) model. The model consists of an economy closed to international trade with public expenditure. As is standard in this literature, private investment $(I)$ and the government expenditure $(G)$ are exogenously given, whereas consumption $(C)$ is comprised of two components: the former component depends (linearly) on aggregate income $(Y)$. The latter one is independent of income (autonomous component) and it can be interpreted as the part of aggregate consumption explained, for instance, by wealth and interest rate $(\bar{C})$. The static model is detailed as follows:

$$
\begin{gathered}
D=C+I+G, \\
C=\bar{C}+c Y, \quad 0<c<1, \\
I=\bar{I}, \\
G=\bar{G},
\end{gathered}
$$

where $0<c<1$ is the marginal propensity to consume, and both the private investment and government expenditure are constant and fixed at $\bar{I}$ and $\bar{G}$, respectively. Equilibrium is obtained based on the following equality between aggregate demand and aggregate supply in the goods market, that is:

$$
D=Y \text {. }
$$

Let us now turn to a continuous-time dynamic setup (with fixed delays) by considering that aggregate consumption at a generic time $t$ depends on income at a preceding date $t-\tau_{c}\left(\tau_{c} \geq 0\right)$, that is $C(t)=\bar{C}+c Y\left(t-\tau_{c}\right)$, and that private investments and the government expenditure are constant and fixed at $G(t)=\bar{G}$ and $I(t)=\bar{I}$, respectively. In addition, the goods market does not necessarily clear thus generating a positive or negative excess demand at every time $t$, that is $Z(t)=D(t)-Y(t)$. There also exists an adjustment mechanism reacting at such a disequilibrium with a certain time lag $\tau_{a} \geq 0$, that is $Z\left(t-\tau_{a}\right)$. This adjustment mechanism may take two distinct forms:

$$
\dot{Y}(t)=\gamma g\left(Z\left(t-\tau_{a}\right)\right)
$$

and

$$
\frac{\dot{Y}(t)}{Y(t)}=\gamma g\left(Z\left(t-\tau_{a}\right)\right)
$$

where $\gamma>0$ is a measure of the reactivity of the adjustment mechanism to a disequilibrium in the goods market. The former mechanism models out an instantaneous change in the level of income as a reaction to a disequilibrium in the goods market. The latter mechanism, instead, measures a variation in the rate of change in income. In what follows, we will consider a model that incorporates a classical linear version of $g$, that is

$$
g\left(Z\left(t-\tau_{a}\right)\right)=Z\left(t-\tau_{a}\right),
$$

and a model that incorporates a nonlinear version of $g$ as in Naimzada and Pireddu (2014a), that is

$$
g\left(Z\left(t-\tau_{a}\right)\right)=\widetilde{g}\left(Z\left(t-\tau_{a}\right)\right):=a_{2}\left[\frac{a_{1}+a_{2}}{a_{1} e^{-Z\left(t-\tau_{a}\right)}+a_{2}}-1\right]
$$


where $a_{1}>0$ and $a_{2}>0$. In the nonlinear case (9), $g$ is an increasing function and $g(0)=0$. Moreover, it is bounded from below by $-a_{2}$ and from above by $a_{1}$, as is noted by Naimzada and Pireddu (2014a). Therefore, in contrast to the linear case (8), income variations in (9) are gradual. This allows to prevent the real market from diverging and it may cause fluctuations in real variables (income and employment), as it will be shown in Section 6 devoted to numerical simulations. By combining the different types of dynamic mechanisms detailed in (6) and (7) with the two definitions of $g$ provided in (8) and (9), we get the following four delay differential equations systems:

$$
\begin{aligned}
\dot{Y}(t) & =\gamma\left[A+c Y\left(t-\tau_{1}\right)-Y\left(t-\tau_{2}\right)\right], \\
\dot{Y}(t) & =\gamma Y(t)\left[A+c Y\left(t-\tau_{1}\right)-Y\left(t-\tau_{2}\right)\right], \\
\dot{Y}(t) & =a_{2} \gamma\left[\frac{a_{1}+a_{2}}{a_{1} e^{-\left[A+c Y\left(t-\tau_{1}\right)-Y\left(t-\tau_{2}\right)\right]}+a_{2}}-1\right], \\
\dot{Y}(t) & =a_{2} \gamma Y(t)\left[\frac{a_{1}+a_{2}}{a_{1} e^{-\left[A+c Y\left(t-\tau_{1}\right)-Y\left(t-\tau_{2}\right)\right]}+a_{2}}-1\right],
\end{aligned}
$$

where we have defined $A:=\bar{C}+\bar{G}+\bar{I}$ as a constant collecting all the autonomous components of the aggregate demand and $\tau_{1}:=\tau_{a}+\tau_{c}$ and $\tau_{2}:=\tau_{a}$, so that $\tau_{1} \geq \tau_{2}$. We recall that $\tau_{c}$ represents a parameter that captures the time delay with which current consumption reacts to past production, whereas $\tau_{a}$ is the time delay that measures the reaction of the adjustment mechanism to a positive or negative excess demand in the goods market. Eqs. (10)-(13) have exactly the same positive equilibrium point $Y_{*}:=A /(1-c)$ obtained by imposing $Y\left(t-\tau_{1}\right)=$ $Y\left(t-\tau_{2}\right)=Y(t)$ and $\dot{Y}(t)=0$. In particular, when $\tau_{1}=\tau_{2}=0, Y_{*}$ is globally asymptotically stable for every initial condition $Y(0)>0$.

The next two sections study local stability, the nature of the Hopf bifurcation and provide some global stability results in the two distinct cases $\tau_{1}>\tau_{2}$ and $\tau_{1}=\tau_{2}$.

\section{Case $\tau_{1}>\tau_{2}$}

In this section we assume that $\tau_{1}>\tau_{2}$. In this case, aggregate consumption reacts based on aggregate income prevailing in a previous period with a time lag $\tau_{c}$. To study local stability and the nature of the Hopf bifurcation, we linearize Eqs. (10)-(13) around $Y_{*}$ and get

$$
\dot{Y}(t)=-A_{1}\left[Y\left(t-\tau_{1}\right)-Y_{*}\right]-A_{2}\left[Y\left(t-\tau_{2}\right)-Y_{*}\right],
$$

where

$$
\begin{aligned}
& A_{1}=-\gamma c<0, \quad A_{2}=\gamma>0 \quad \text { for Eq. (10), } \\
& A_{1}=-\gamma c Y_{*}<0, \quad A_{2}=\gamma Y_{*}>0 \quad \text { for Eq. (11), } \\
& A_{1}=-\frac{a_{1} a_{2} \gamma c}{a_{1}+a_{2}}<0, \quad A_{2}=\frac{a_{1} a_{2} \gamma}{a_{1}+a_{2}}>0 \quad \text { for Eq. (12), } \\
& A_{1}=-\frac{a_{1} a_{2} \gamma c Y_{*}}{a_{1}+a_{2}}<0, \quad A_{2}=\frac{a_{1} a_{2} \gamma Y_{*}}{a_{1}+a_{2}}>0 \quad \text { for Eq. (13). }
\end{aligned}
$$


We note that regardless of the model used, $A_{1}$ is decreasing with respect to $c$ and $A_{2}$ is increasing with respect to $\gamma$. The characteristic equation of (14) is as follows

$$
\lambda=-A_{1} e^{-\lambda \tau_{1}}-A_{2} e^{-\lambda \tau_{2}} .
$$

In the particular case $\tau_{2}=0$, the characteristic equation (15) reduces to

$$
\lambda=-A_{1} e^{-\lambda \tau_{1}}-A_{2} .
$$

Let $\lambda=i \omega$ be a purely imaginary root of this equation. Separating real and imaginary parts, $\omega$ satisfies

$$
\omega=A_{1} \sin \omega \tau_{1}, \quad A_{2}=-A_{1} \cos \omega \tau_{1},
$$

yielding $\omega^{2}=A_{1}^{2}-A_{2}^{2}<0$ as $c<1$. Hence, we can conclude that if $\tau_{2}=0$ then $Y_{*}$ is locally asymptotically stable for any $\tau_{1}>0$. By continuity, for sufficiently small $\tau_{2}>0, Y_{*}$ remains locally asymptotically stable. Next, choose $\tau_{2}>0$ as a bifurcation parameter. If $\lambda=i \omega$ (with $\omega>0)$ is a solution of (15) then we must have

$$
i \omega=-A_{1} e^{-i \omega \tau_{1}}-A_{2} e^{-i \omega \tau_{2}} .
$$

Separating real and imaginary parts, we have

$$
\omega-A_{1} \sin \left(\omega \tau_{1}\right)=A_{2} \sin \left(\omega \tau_{2}\right), \quad A_{1} \cos \left(\omega \tau_{1}\right)=-A_{2} \cos \left(\omega \tau_{2}\right) .
$$

By direct calculation, we obtain

$$
\sin \left(\omega \tau_{1}\right)=\frac{\omega^{2}+A_{1}^{2}-A_{2}^{2}}{2 \omega A_{1}} .
$$

For any $\tau_{1}>0$, this equation has a finite number of positive zeros $\omega_{j}, j=1,2, \ldots, m$. It is clear that for every arbitrary chosen $\tau_{1}>0$ and for each $\omega_{j}$ there exists an infinite number of $\tau_{2}$ such that $A_{1} \cos \left(\omega_{j} \tau_{1}\right)=-A_{2} \cos \left(\omega_{j} \tau_{2}\right)$. For all $j=1,2, \ldots, m$, define

$$
\tau_{2}^{j}=\min \left\{\tau_{2}>0: A_{1} \cos \left(\omega_{j} \tau_{1}\right)=-A_{2} \cos \left(\omega_{j} \tau_{2}\right)\right\},
$$

and set

$$
\bar{\tau}_{2}^{0}=\frac{\tau_{2}^{0}}{\left|A_{1}\right|}
$$

where $\tau_{2}^{0}=\min \left\{\tau_{2}^{j}: j=1,2, \ldots, m\right\}$.

Theorem 1 Let $\bar{\tau}_{2}^{0}$ be as in (16). If $\tau_{1} \in\left(0, \pi / 2 \sqrt{A_{2}^{2}-A_{1}^{2}}\right)$, then the non-trivial equilibrium $Y_{*}$ to Eqs. (10)-(13) is locally asymptotically stable for $\tau_{2} \in\left[0, \bar{\tau}_{2}^{0}\right)$ and the Hopf bifurcation occurs for $\tau_{2}=\bar{\tau}_{2}^{0}$.

Proof. The proof can be found in Piotrowska (2007) by looking at the case $A_{1}<0, A_{2}>0$ and $A_{2} /\left|A_{1}\right|=1 / c>1$.

We note that under some strong assumptions on the size of $\tau_{2}$, it is possible to obtain a global stability $^{5}$ result regardless of the value of $\tau_{1}$. In the case of model (10), we have the following result.

\footnotetext{
${ }^{5}$ The result about global stability actually holds on a set of initial functions wider than that defined in Theorem 2. This is because the hypotheses of the theorem are set based on the economic meaning of variable $Y(t)$.
} 
Theorem 2 Assume that $\tau_{2}<1 /(\gamma e)$. Then the steady-state solution of Eq. (10) is globally asymptotically stable for any initial function $Y(t)$ positive in the interval $\left[-\tau_{1}, 0\right]$.

Proof. Let $x=Y-Y_{*}$. Then Eq. (10) becomes $\dot{x}(t)=-\gamma x\left(t-\tau_{2}\right)+\gamma c x\left(t-\tau_{1}\right)$ with $x_{*}=0$. The statement follows from Theorem 4.2. in Györi (1990) with $d=\gamma, \tau=\tau_{2}, \sigma=\tau_{1}$, $f(x(t-\sigma))=\gamma c x\left(t-\tau_{1}\right)$, by noting that $|f(x)|<d|x|$ for $x \neq 0$ holds true being $c<1$.

This means that if the adjustment mechanism of the excess demand is quite fast, the stationary state equilibrium is stable even starting from initial conditions far enough from the stationary equilibrium, and this holds regardless of the formation mechanism of aggregate consumption.

\section{Case $\tau_{1}=\tau_{2}=\tau\left(\tau_{c}=0\right)$}

The results of the previous section simplify when, similarly to Naimzada and Pireddu (2014a), one assumes that there exists no time lag on the formation mechanism of aggregate consumption $\left(\tau_{c}=0\right)$, that is when one considers that aggregate consumption at time $t$ depends on aggregate production prevailing in the same period. In this case, Eqs. (10)-(13) become

$$
\begin{aligned}
\dot{Y}(t) & =\gamma[A-(1-c) Y(t-\tau)] \\
\dot{Y}(t) & =\gamma Y(t)[A-(1-c) Y(t-\tau)] \\
\dot{Y}(t) & =a_{1} a_{2} \gamma\left[\frac{1-e^{-[A+(c-1) Y(t-\tau)]}}{a_{1} e^{-[A+(c-1) Y(t-\tau)]}+a_{2}}\right]=a_{1} a_{2} \gamma f(Y(t-\tau)), \\
\dot{Y}(t) & =a_{1} a_{2} \gamma Y(t)\left[\frac{1-e^{-[A+(c-1) Y(t-\tau)]}}{a_{1} e^{-[A+(c-1) Y(t-\tau)]}+a_{2}}\right]=a_{1} a_{2} \gamma Y(t) f(Y(t-\tau)) .
\end{aligned}
$$

It is immediate that Eqs. (17)-(20) have the unique positive equilibrium $Y_{*}=A /(1-c)$. The linearization of Eqs. (17)-(20) at $Y=Y_{*}$ is

$$
\dot{Y}(t)=M\left[Y(t-\tau)-Y_{*}\right]
$$

where

$$
\begin{aligned}
& M=\gamma(c-1)<0 \text { for Eq. (17), } \\
& M=\gamma(c-1) Y_{*}<0 \text { for Eq. (18), } \\
& M=\frac{a_{1} a_{2} \gamma(c-1)}{a_{1}+a_{2}}<0 \text { for Eq. (19), } \\
& M=\frac{a_{1} a_{2} \gamma(c-1) Y_{*}}{a_{1}+a_{2}}<0 \text { for Eq. (20). }
\end{aligned}
$$

Its corresponding characteristic equation is given by $\lambda=M e^{-\lambda \tau}$. For $\omega>0, \lambda=i \omega$ is a root of this equation if $i \omega=M e^{-i \omega \tau}$. Hence, we obtain

$$
\omega=-M \sin \omega \tau, \quad 0=M \cos \omega \tau,
$$


which lead to $\omega=\omega_{0}=-M$ and $\tau=\tau_{0}=\pi /\left(2 \omega_{0}\right)$. Let $\lambda(\tau)=\alpha(\tau)+i \omega(\tau)$ denote a root of Eq. (21) near $\tau=\tau_{0}$ such that $\alpha\left(\tau_{0}\right)=0, \omega\left(\tau_{0}\right)=\omega_{0}$. Differentiating both sides of the characteristic equation in (21) with respect $\tau$ gives

$$
\left(\frac{d \lambda}{d \tau}\right)^{-1}=-\frac{1}{\lambda^{2}}-\frac{\tau}{\lambda}
$$

so that we get

$$
\operatorname{Re}\left(\frac{d \lambda}{d \tau}\right)_{\tau=\tau_{0}}^{-1}=\frac{1}{\omega_{0}^{2}}>0, \quad \text { i.e. }\left.\frac{d(\operatorname{Re} \lambda)}{d \tau}\right|_{\tau=\tau_{0}}>0
$$

yielding that the root $\lambda(\tau)$ of Eq. (21) near $\tau_{0}$ crosses the imaginary axis from the left to the right. It is immediately seen that $\lambda=i \omega$ is a simple root of (21). Thus, we arrive at the following conclusion.

Theorem 3 The non-trivial equilibrium $Y_{*}$ to Eqs. (17)-(20) is locally asymptotically stable for $\tau \in[0,-\pi /(2 M))$ and the Hopf bifurcation occurs for $\tau=-\pi /(2 M)$.

The following theorem characterizes the global behaviour ${ }^{6}$ of the nontrivial equilibrium $Y_{*}$.

Theorem 4 1. Assume that $\tau<3 /[2(1-c) \gamma]$. Then the positive steady state solution of Eq. (17) is globally asymptotically stable for any initial function $Y(t)$ positive in the interval $[-\tau, 0]$.

2. Assume that $\tau<3 /(2 A \gamma)$. Then the positive steady state solution of Eq. (18) is globally asymptotically stable for any initial function $Y(t)$ positive in the interval $[-\tau, 0]$.

Proof. 1) Let $x=Y-Y_{*}$. Then Eq. (17) becomes $\dot{x}(t)=-\gamma(1-c) x(t-\tau)$ with $x_{*}=0$. The proof can be found in Yorke (1970). 2) Rewriting (18) as $Y(t)=(\gamma A) Y(t)\left[1-Y(t-\tau) / Y_{*}\right]$, our equation becomes the Hutchinson's equation. Using the transformation $x=Y(\tau t) / Y_{*}-1$, and letting $a=\gamma A \tau$, leads to the classical Wright's equation $\dot{x}(t)=-a x(t-1)[1+x(t)]$. The statement follows from Wright (1955).

In this case, we are able to give a global stability result also in the nonlinear model described by (18).

\section{$5 \quad$ Stability switching curves}

As is known, the study of the dynamic (just focusing on local) properties of a system with two distinct delays is so far under scrutiny in the mathematical literature (see Bi and Ruan, 2013 for recent results on codimension-two bifurcations). Therefore, in this section we concentrate only on the analysis of some properties of dynamic systems (10)-(13) that can be of importance from an economic point of view. In order to understand how time delays actually affect stability outcomes in the models discussed above, we will apply the techniques developed by Gu et al.

\footnotetext{
${ }^{6}$ See Footnote 5 for the definition of the set of initial conditions.
} 
(2005). Given these results, it is possible to define (by fixing the other parameters of the model) the set of delays $\left(\tau_{1}, \tau_{2}\right)$ such that the system is locally asymptotically stable.

Starting from the characteristic equation (15), we define the following polynomials:

$$
\begin{gathered}
p_{0}(\lambda)=\lambda, \\
p_{1}(\lambda)=A_{1}, \\
p_{2}(\lambda)=A_{2} .
\end{gathered}
$$

The zeros of (15) coincide with the zeros of

$$
B\left(\lambda, \tau_{1}, \tau_{2}\right)=1+B_{1}(\lambda) e^{-\tau_{1} \lambda}+B_{2}(\lambda) e^{-\tau_{2} \lambda},
$$

where $B_{j}=p_{j}(\lambda) / p_{0}(\lambda), j=1,2$. Following Gu et al. (2005), the fist step to approach the problem of stability of the stationary equilibrium is to consider the three terms on the left-handside of $B\left(\lambda, \tau_{1}, \tau_{2}\right)=0$, that is $1, B_{1}(\lambda) e^{-\tau_{1} \lambda}$ and $B_{2}(\lambda) e^{-\tau_{2} \lambda}$ as three vectors in the complex plane.

Now, we have to identify the set $\Omega$ of $\omega$ that fulfils the conditions such that complex conjugate roots do exist and, from a geometrical point of view, it consists of characterising conditions such that the vectors above form a triangle (Figure 1). Then, by introducing

$$
\begin{gathered}
L_{1}(\omega):=\left|B_{1}(i \omega)\right|+\left|B_{2}(i \omega)\right|=\frac{A_{2}-A_{1}}{\omega}, \\
L_{2}(\omega):=\left|B_{1}(i \omega)\right|-\left|B_{2}(i \omega)\right|=\frac{-A_{1}-A_{2}}{\omega}
\end{gathered}
$$

it is sufficient to identify $\Omega$ as the sets such that the graph of function $L_{2}$ belongs to region $G:=\{(\omega, z): z \in[-1,1]\}$, whereas the graph of function $L_{1}$ does not.

By using the law of the cosine, it is possible to get the internal angles $\theta_{1}, \theta_{2} \in[0, \pi]$ formed by vectors (see the triangle in Figure 1), that is

$$
\theta_{1}=\cos ^{-1}\left(\frac{1+\left|B_{1}(i \omega)\right|^{2}-\left|B_{2}(i \omega)\right|^{2}}{2\left|B_{1}(i \omega)\right|}\right)
$$

and

$$
\theta_{2}=\cos ^{-1}\left(\frac{1+\left|B_{2}(i \omega)\right|^{2}-\left|B_{1}(i \omega)\right|^{2}}{2\left|B_{2}(i \omega)\right|}\right)
$$

and then identify solutions of $B\left(\omega i, \tau_{1}, \tau_{2}\right)=0$ as the locus of points in $\left(\tau_{1}, \tau_{2}\right)$ plane drawn by the following curves parametrized with respect to $\omega$ :

$$
\begin{cases}\tau_{1}=\tau_{1}^{v_{1}^{ \pm}}(\omega)=\frac{\arg \left(B_{1}(i \omega)\right)+\left(2 v_{1}-1\right) \pi \pm \theta_{1}}{\omega} \geq 0, & v_{1}=v_{1,0}^{ \pm}, v_{1,0}^{ \pm}+1, v_{1,0}^{ \pm}+2, \ldots \\ \tau_{2}=\tau_{2}^{v_{2}^{ \pm}}(\omega)=\frac{\arg \left(B_{2}(i \omega)\right)+\left(2 v_{2}-1\right) \pi \mp \theta_{2}}{\omega} \geq 0, & v_{2}=v_{2,0}^{ \pm}, v_{2,0}^{ \pm}+1, v_{2,0}^{ \pm}+2, \ldots\end{cases}
$$

where $v_{1,0}^{+}, v_{1,0}^{-}, v_{2,0}^{+}$and $v_{2,0}^{-}$are the smallest possible integers (that may be negative and may depend on $\omega$ ) such that the corresponding calculated values of $\tau_{1}^{v_{1,0}^{+}}, \tau_{1}^{v_{1,0}^{-}}, \tau_{2}^{v_{2,0}^{+}+}$and $\tau_{2}^{v_{2,0}^{-}-}$ are non-negative.

We note that in this case, graphs of $L_{1}(\omega)$ and $L_{2}(\omega)$ are branches of hyperbolas. Then, the following proposition holds. 
Proposition 1 For any $A_{1}$ and $A_{2}$ there exists an interval $\Omega=\left(A_{1}+A_{2}, A_{2}-A_{1}\right)$ of type 2 , 3 (this follows the notation introduced by $G u$ et al., 2005) and the stability switching curves are spiral-like curves with horizontal axes.

Proof. The proof follows from Proposition 4.5 (Gu et al., 2005, p. 243).

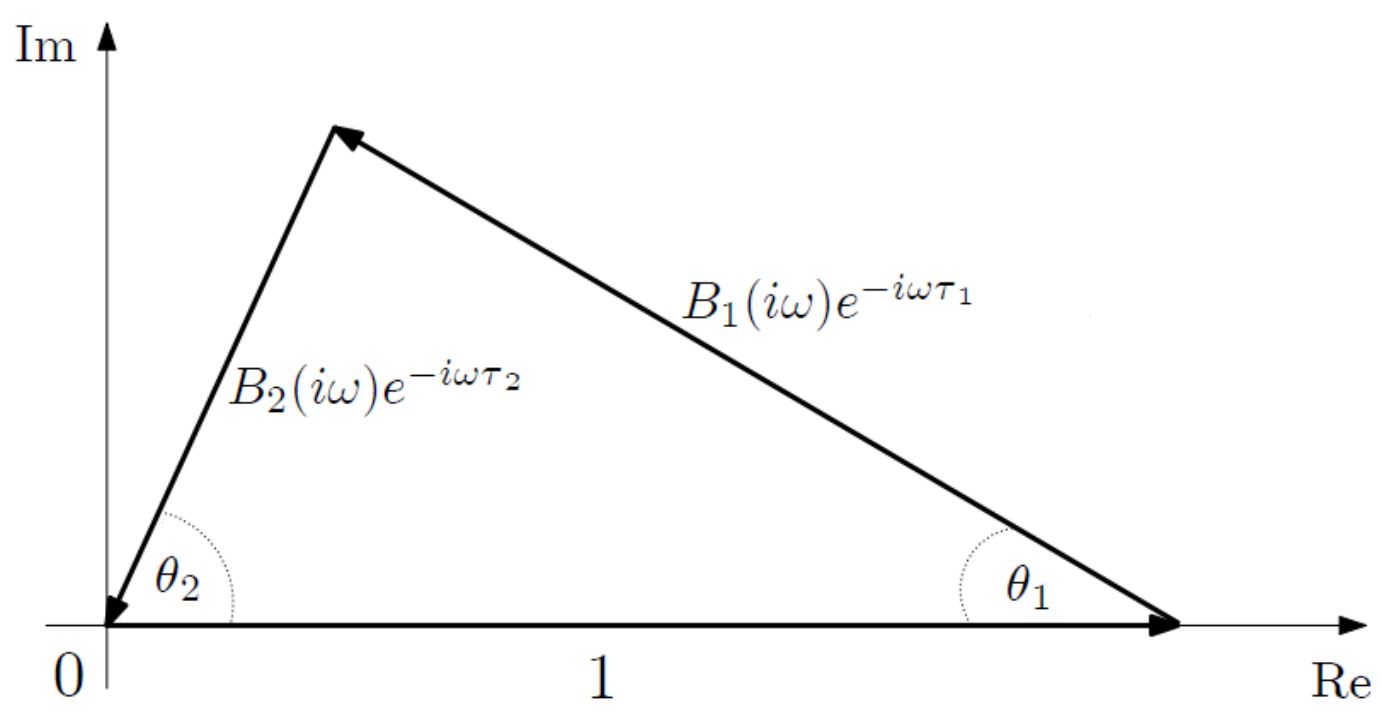

Figure 1. Triangle formed by $1,\left|B_{1}(i \omega)\right|$ and $\left|B_{2}(i \omega)\right|$.

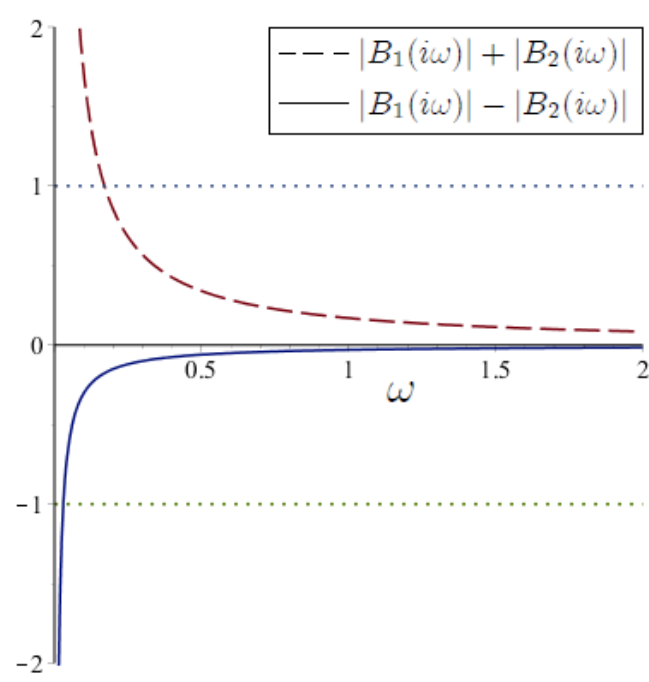

(a)

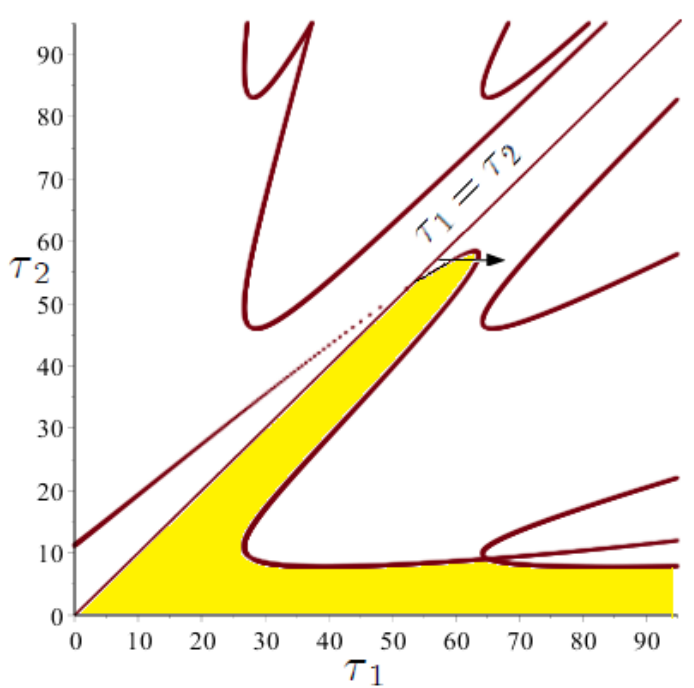

(b) 


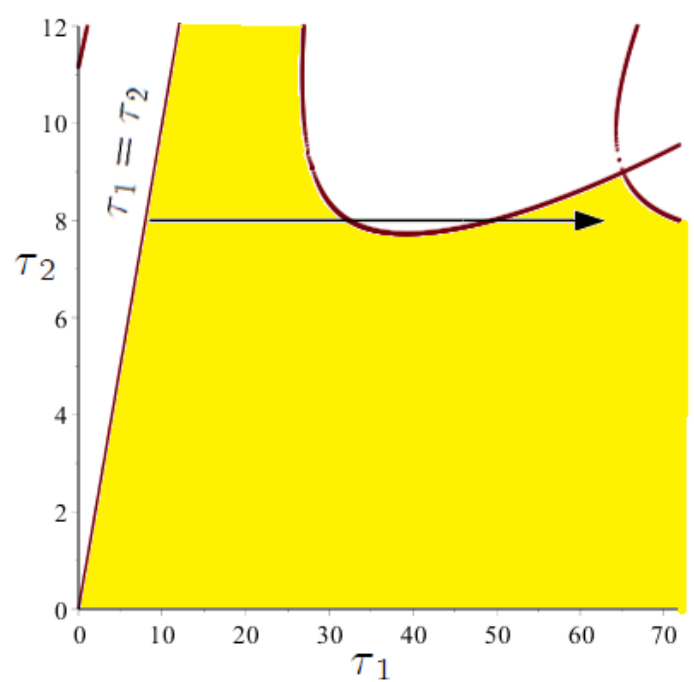

(c)

Figure 2. Parameter set: $A=12, c=0.7, A_{1}=-0.07$ and $A_{2}=0.1$. The stationary state value of income is $Y_{*}=40$. In the linear version (10) of the model, the values of $A_{1}$ and $A_{2}$ are obtained by setting $\gamma=0.1$; in the nonlinear logistic version (11) of the model, the values of $A_{1}$ and $A_{2}$ are obtained by setting $\gamma=0.0025$; in the case of model (12) with a nonlinear adjustment mechanism in the level of income, the values of $A_{1}$ and $A_{2}$ are obtained by setting $a_{1}=1.5, a_{2}=3$ and $\gamma=0.1$; in the case of model (13) with a nonlinear adjustment mechanism in the rate of change in income, the values of $A_{1}$ and $A_{2}$ are obtained by setting $a_{1}=1.5$, $a_{2}=3$ and $\gamma=0.0025$. (a) $\left|B_{1}(i \omega)\right| \pm\left|B_{2}(i \omega)\right|$ versus $\omega$. Set $\Omega$ consists of a unique interval $\Omega=(0.03,0.17)$. (b) Spiral-like stability switching curves with horizontal axes in $\left(\tau_{1}, \tau_{2}\right)$ plane. $^{7}$ The region below the $45^{\circ}$ line defines the set of couples of feasible delays $\left(\tau_{1}>\tau_{2}\right)$. The yellow area shows a portion of the stability region in $\left(\tau_{1}, \tau_{2}\right)$ plane. The figure highlights the case of instability-stability-instability of the stationary state equilibrium (see the arrow in the figure) also showing the existence of a corridor of stability. (c) Enlargement view of Panel (b); for lower values of $\tau_{2}$ the exists a case of stability-instability-stability of the stationary state equilibrium (see the arrow in the figure).

Panel (a) of Figure 2 depicts $L_{1}(\omega)$ and $L_{2}(\omega)$ for all the dynamic models detailed above. The yellow region depicted in Panel (b) of Figure 2 shows the couples $\left(\tau_{1}, \tau_{2}\right)$ such that the stationary equilibrium $Y_{*}$ of systems (10)-(13) is locally asymptotically stable. By looking at this panel we can deduce some results that can be of interest from an economic point of view. In line with the results discussed in Section 3, it is clear that for small values of $\tau_{2}$ the value of $\tau_{1}$ can indefinitely grow without affecting the local stability of the stationary equilibrium (corridor of stability). This means that when aggregate production reacts quickly to the excess demand, the economic system converges towards the stationary equilibrium regardless of the timing that (current) aggregate consumption needs to react to aggregate production. With regard to Panel (b) of Figure 2, we note that by fixing $\tau_{2}=57$ and letting $\tau_{1}$ vary from 57 onwards it is possible to move from a configuration of time delays for which the stationary state value of income is unstable $\left(\tau_{1}<59.6\right)$ to a region in which it is stable $\left(59.6<\tau_{1}<63.5\right)$ to eventually end up in an area of instability (this holds when $\tau_{1}>63.5$ ). In contrast, Panel (c) of Figure 2 shows

\footnotetext{
${ }^{7}$ For a complete characterisation of stability switching curves in an economic model (a cobweb model with heterogeneous producers), see the recent study of Gori et al. (2015).
} 
the opposite situation. In particular, by fixing $\tau_{2}=8$ and letting $\tau_{1}$ vary in the range $[30,60]$ we move from a configuration of time delays for which the stationary state value of income is stable $\left(\tau_{1}<32\right)$ to a region in which the stationary state value is unstable $\left(32<\tau_{1}<51.5\right)$, to eventually get a stable stationary state value of income when $\tau_{1}>51.5$. The possibility of moving from a case of initial (i.e., $\tau_{1} \in[0, \tilde{\tau}]$ ) instability to stability, which is uncommon in single-delay models (and also in systems with delay-dependent parameters, as analysed by He et al., 2009, and $\mathrm{He}$ and $\mathrm{Li}, 2012$ ), is most commonly encountered in multiple-delay models, as is shown in the works of Gori et al. (2015). With specific regard to Figure 2(b), when consumers are backward looking enough, a goods market reacting to an aggregate demand disequilibrium either too slowly or quickly leads to instability. Only time of reaction belonging to a specific (intermediate) interval can lead to the stability of the stationary equilibrium.

\section{Numerical simulations}

The techniques proposed by Gu et al. (2005) have allowed to characterise the stability regions for general dynamic systems with two discrete delays, but they have not been able, for instance, to infer about the timing with which a typical trajectory approaches a stationary state equilibrium and what actually happens when the stationary solution loses stability. In order to fill this gap here, we resort to some numerical exercises. To this purpose, Figure 3 shows different dynamics generated by the linear adjustment mechanism (8) when (ceteris paribus with regard to parameter values and initial conditions that guarantee that the system is asymptotically stable) one considers a continuous-time system with fixed delays (blue solid line), a continuous-time system without delays (black dashed line) and a discrete-time map (red dotted line). We note that the continuous-time system produces monotonic dynamics (by setting the values of time delays to zero one gets a uni-dimensional dynamic system) but the corresponding discrete-time map and the continuous-time system with delays generate non-monotonic dynamics. However, the fluctuations produced by the delayed continuous-time system tend to stabilise around the stationary-state equilibrium more slowly than those produced by the discrete-time map. By looking at Figure 3, in fact, one can observe that after around the thirtieth iterate the time series generated by the discrete-time model becomes almost identical to the level of the stationary-state equilibrium, whereas after around $t=100$ the time series generated by the continuous-time model with time delays still shows important fluctuations. 


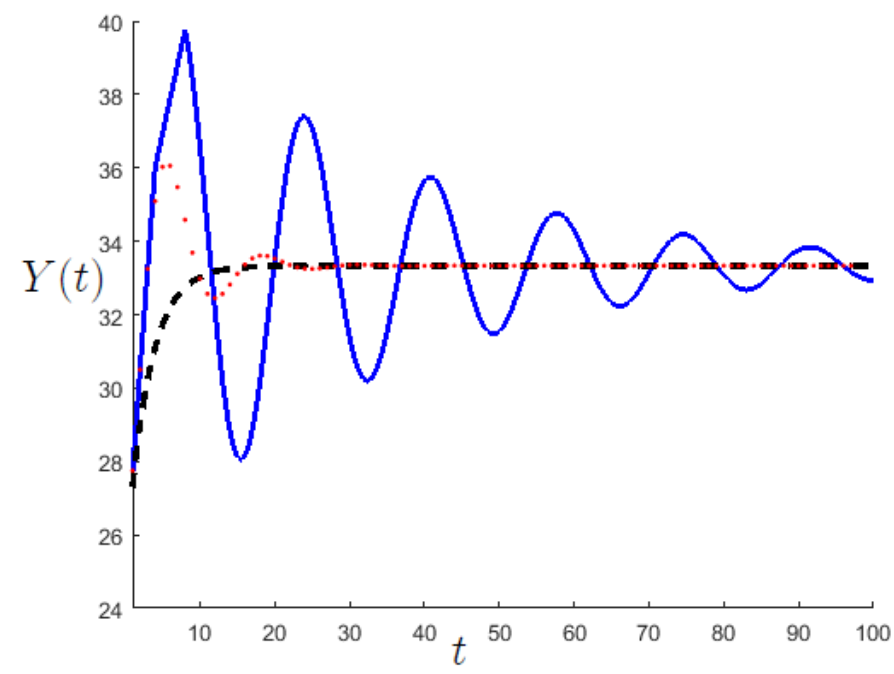

Figure 3. The adjustment mechanism is linear in the instantaneous change in the level of income. Different trajectories converging towards the stationary state equilibrium generated by the same initial condition depending on whether dynamics are described by a continuous-time system with fixed delays (blue solid line), a continuous-time system without delays (black dashed line) and a discrete-time map (red dotted line). Parameter set: $A=10, c=0.7, \gamma=1.1, \tau_{1}=$ $\tau_{2}=3$ and $Y(0)=25$. In this case, the discrete-time system is $Y_{t+1}=Y_{t}+\gamma[A-(1-c) Y(t-2)]$.

When the adjustment mechanism is linear in the instantaneous change in the level of income, the model does not produce interesting dynamics when the stationary state equilibrium loses stability. This is because, for several parametric specifications, the system tends to diverge when the fixed point is unstable. In contrast, it is possible to observe more interesting dynamic outcomes when the rate of change in income $(\dot{Y}(t) / Y(t))$ is linear with respect to the excess demand $Z\left(t-\tau_{a}\right)$. In this case, in fact, as is well known by the studies on the Hutchinson's equation (see Ruan, 2006 for a survey), when the stationary state equilibrium loses stability the system undergoes a super-critical Hopf bifurcation, so that - after a sufficiently long transient - the typical trajectory convergent towards the attracting limit cycle is characterised by the existence of a unique maximum value and a unique minimum value. Other phenomena are possible by increasing one of the two time delays further. In particular, the attractor can change its geometry ${ }^{8}$ and the long-term dynamics of a typical trajectory convergent towards the attractor of the system can be characterised by the existence of several local maximum and minimum values, whilst never becoming a chaotic attractor. This phenomenon is illustrated in Figure 4(a). The figure was plotted by letting $\tau_{1}$ vary in the interval $\left[\tau_{2}, 0.18\right]$ with step 0.01 and keeping the other parameters and the initial condition fixed to the values reported in the caption of the figure. Then, for any $\tau_{1}$ we have considered the local maximum and local minimum values of the trajectory so generated after a sufficiently long transient. The values of time delay $\tau_{1}$ for which the fixed point is stable are shown in the figure by a single point ( $\max$ value $=$ min value). After the occurrence of the Hopf bifurcation, the bifurcation diagram shows two points associated with the value of the time delay (one for the maximum and one for the minimum). Due to the change

\footnotetext{
${ }^{8}$ See the work of Braddock and van den Driessche (1983), where the dynamics are studied in the plane of the state variable and its time derivative.
} 
in the geometry of the attractor (as described above), for sufficiently large values of the time delay the bifurcation diagram shows the existence of more than two local maximum and local minimum values. Similar considerations hold for the other bifurcation diagrams in the article. This way of representing bifurcation diagrams is alternative, for instance, to He et al. (2009) and He and $\mathrm{Li}$ (2012), who instead report the set of values taken by the trajectory convergent towards the attractor for each value of the time delay (considered as a bifurcation parameter). The way to represent the bifurcation diagrams used in this work, and often used in both mathematical literature and economic applications, allows to highlight the rise of more complicated dynamics as the bifurcation parameter changes.

We note that for initial conditions far away from the equilibrium, trajectories are divergent also when the system is stable, whereas for too large values of the time delay almost all trajectories diverge. In addition, by comparing the dynamics under linear adjustments in the instantaneous change of the level of income and in the rate of change of income, it is clear that in the latter case the system loses stability for values of time delays lower than those related to the former case (see Figure 3 and Figure 4(b)).

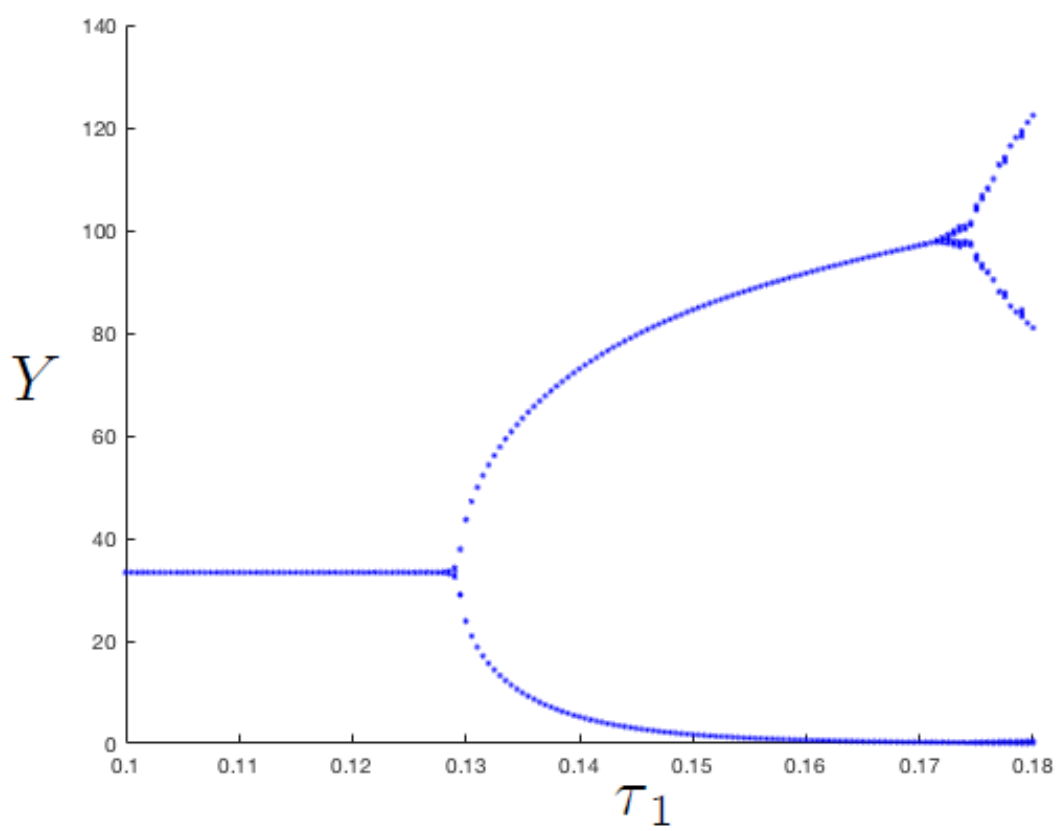

(a) 


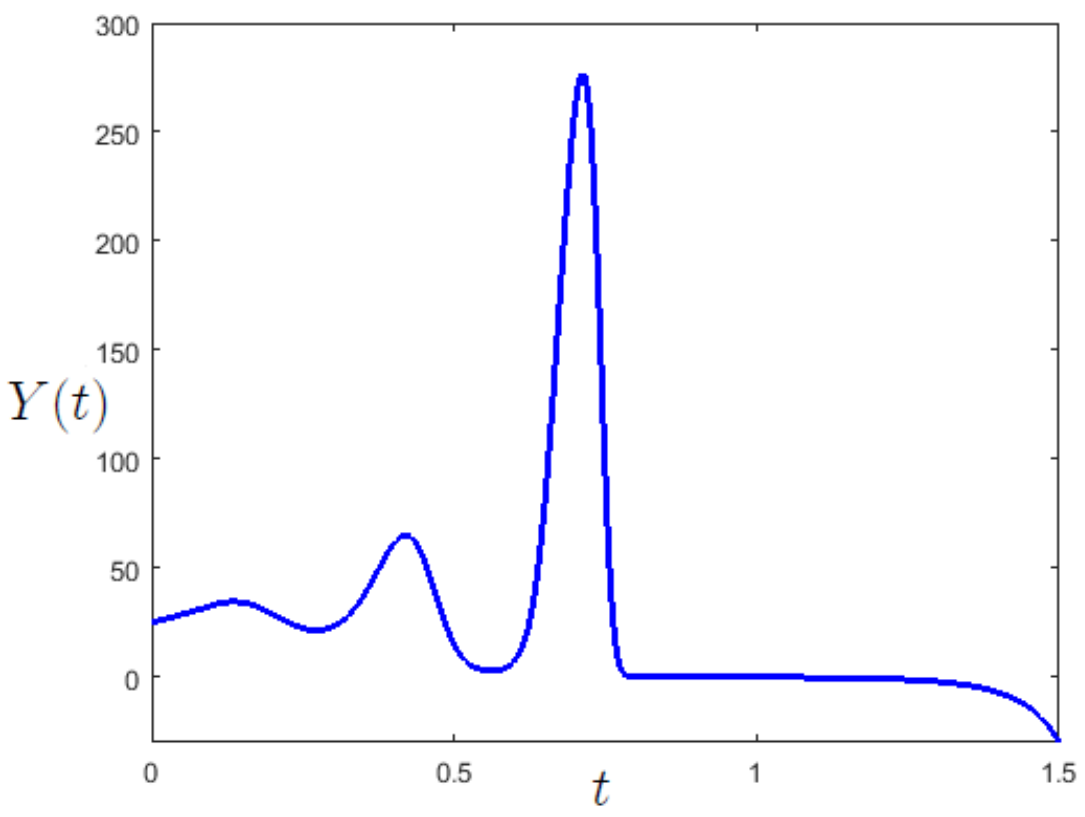

(b)

Figure 4. The adjustment mechanism is linear in the rate of change of income. (a) Bifurcation diagram with respect to $\tau_{1}$. Parameter set: $c=0.7, \gamma=1.1, \tau_{2}=0.1$ and $Y(t)=25$, for $t \in\left[-\tau_{1}, 0\right]$. (b) Divergent trajectory generated by using a value of $\tau_{1}$ slightly larger than 0.18 , that is $\tau_{1}=0.183$.

The problem with divergence is overcome by adopting the mechanism proposed by Naimzada and Pireddu (2014a). The bifurcation diagrams of Figures 5(a) and 5(b), which are respectively related to Panels (b) and (c) of Figure 2, show the (bounded) long-term dynamics of model (12) by considering $\tau_{1}$ as the bifurcation parameter. We note that with this parameter set (in terms of $A_{1}$ and $A_{2}$ ) both the model with linear adjustment in the level of income (model (10)) and the one with linear adjustment in the rate of change in income (model (11)) generate explosive trajectories for some values of $\tau_{1}$. In addition, by considering values of $\tau_{1}$ far enough away from the Hopf bifurcation trajectories show more complicated behaviours, as is shown in Figures 6 (related to model (12)) and 7 (related to model (13)). 


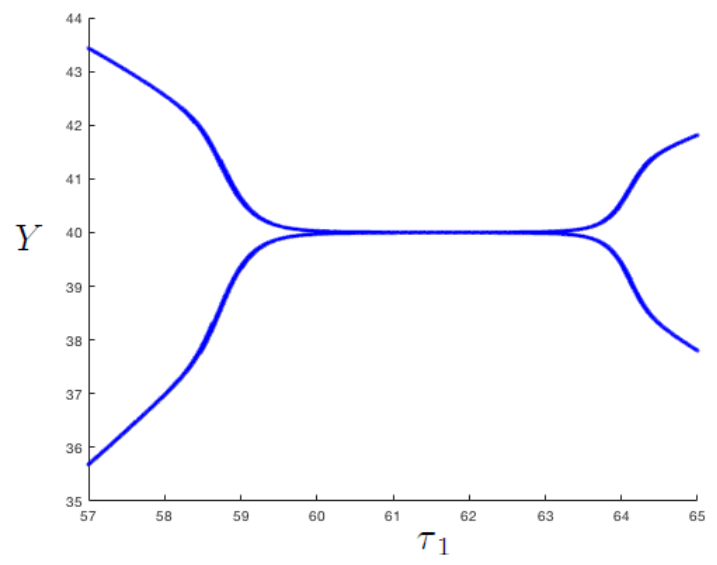

(a)

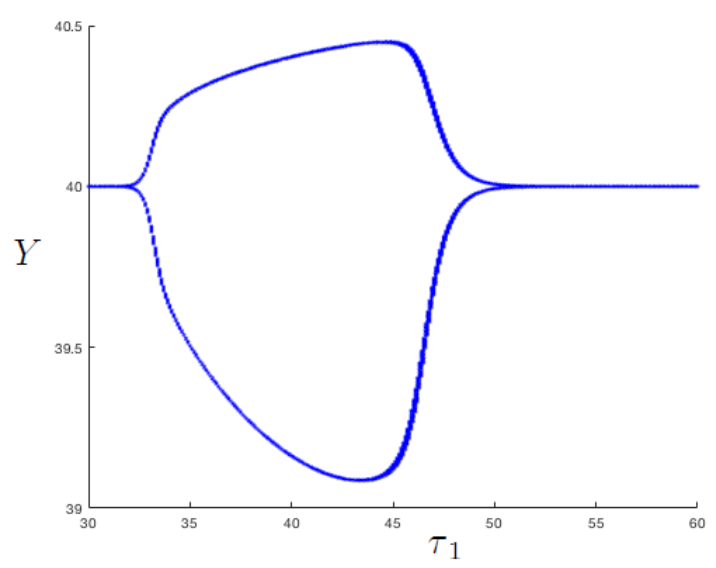

(b)

Figure 5. Bifurcation diagrams for $\tau_{1}$ related to model (12). Parameter set: $A=12, c=0.7$, $\gamma=0.1, a_{1}=1.5$ and $a_{2}=3$. (a) $\tau_{2}=57$, see also Panel (b) of Figure 2 ; (b) $\tau_{2}=8$, see also Panel (c) of Figure 2.

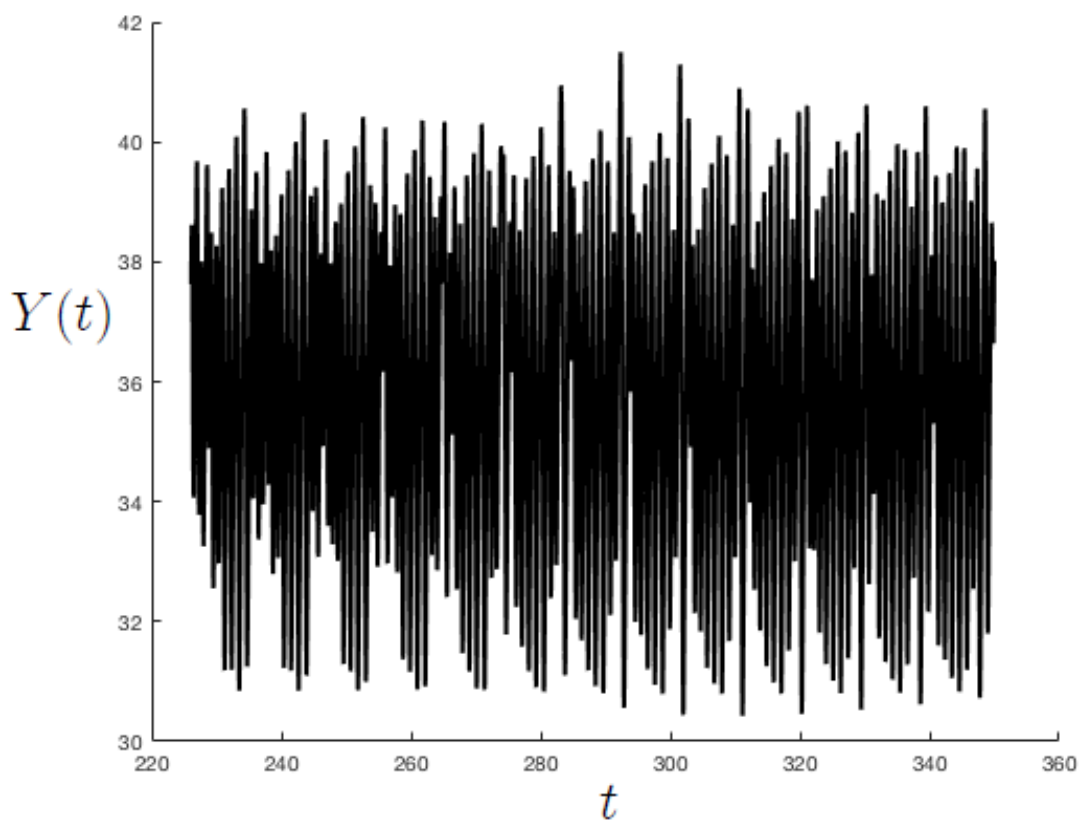

Figure 6. The adjustment mechanism is nonlinear in the instantaneous change in the level of income. Parameter set: $A=12, c=0.7, \gamma=9.1, a_{1}=1.5, a_{2}=3, \tau_{1}=9$ and $\tau_{2}=0.19$. Dynamics generated by the nonlinear mechanism proposed by Naimzada and Pireddu (2014a) when the fixed point is unstable and the system lies in the chaotic region. 


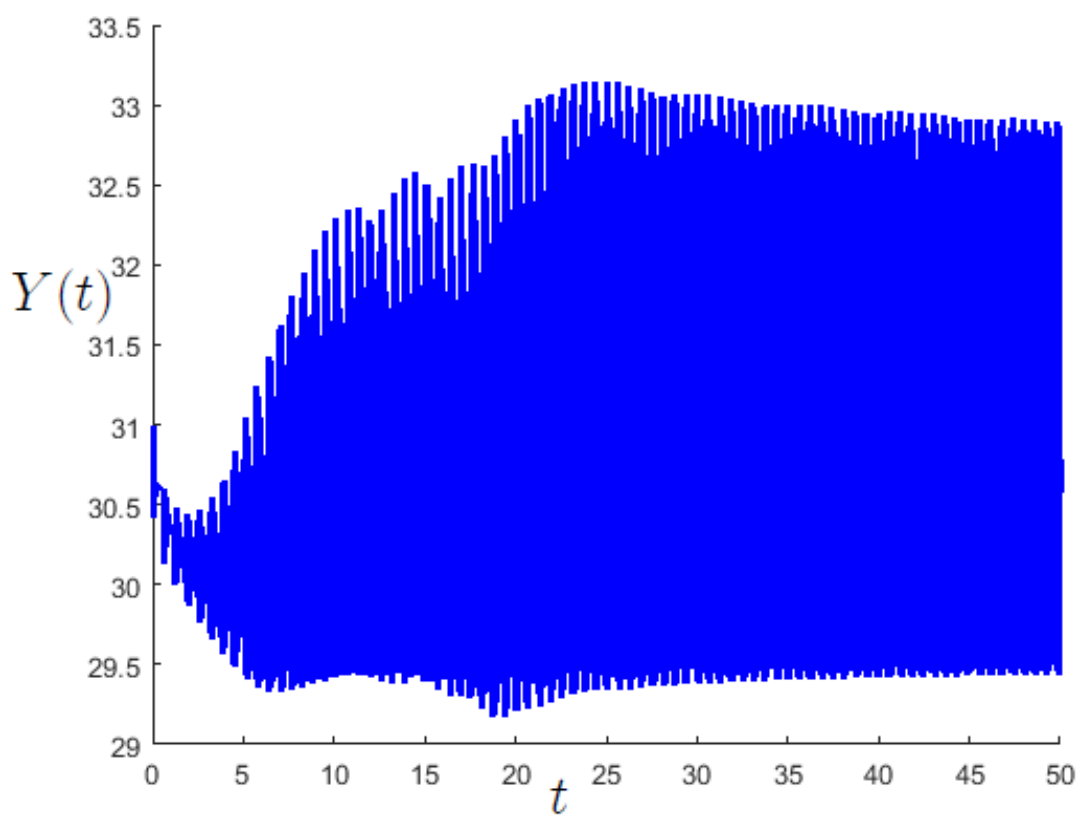

Figure 7. The adjustment mechanism is nonlinear in the rate of change of income. Parameter set: $A=12, c=0.6, \gamma=1, a_{1}=5, a_{2}=2.2, \tau_{1}=0.59$ and $\tau_{2}=0.022$. Dynamics generated by the nonlinear mechanism proposed by Naimzada and Pireddu (2014a) when the fixed point is unstable and the system lies in the chaotic region. It can be noted that with a nonlinear adjustment mechanism based on the rate of change in income the equilibrium is destabilised for smaller values of $\tau_{1}$ than the case of a nonlinear adjustment mechanism based on the instantaneous change in the level of income. The figure shows a typical trajectory convergent towards the $\omega$-limit set.

As is clearly shown in Figures 6 and 7, the dynamics generated by the mechanisms introduced in (12) and (13) imply non-converging trajectories. Now, one can wonder whether the dynamics are periodic, quasi-periodic or chaotic. A formal study about the existence of chaos in models based on delay differential equations has not yet been developed as instead it was in the case of ordinary differential equations or difference equations. Usually, the problem is tackled by using numerical techniques such as the maximum Lyapunov exponent computed from the time series generated by numerical algorithms like the one developed by Wolf et al. (1985) (see also the work of Sprott, 2007). For example, in this case, the attractor depicted in Figure 6 shows a (robust) positive value for the Lyapunov exponent around 0.8, which implies that system (12) is chaotic.

\section{Conclusions}

This work has proposed some modifications of the classical Keynesian macroeconomic model describing goods market dynamics. In comparison with the standard continuous-time (differential equations) version or its discrete-time (difference equations) counterpart, the present article has accounted for a continuous-time model with fixed delays (delay differential equations). The use of delay differential equations instead of differential or difference equations allows to go 
beyond some restrictions of the other modelling approaches in a natural way. The work has provided a complete description of the stability region in the space of time delays by using the stability crossing curves techniques developed by Gu et al. (2005), showing also some results of global stability. It has shown that the extent of time delays is responsible for the existence of interesting dynamic outcomes even when the adjustment mechanism of the excess demand in the goods market is linear (in the level of income or in the rate of change of income). The main aim has been to extend the nonlinear adjustment mechanism proposed by Naimzada and Pireddu (2014a) in a discrete-time setup, showing that its application in a continuous-time setting with fixed delays can provide a starting point for building on more sophisticated models.

Conflict of Interest The authors declare that they have no conflict of interest.

Acknowledgements The authors are grateful to MDEF 2016 conference participants held at University of Urbino, Italy, for valuable comments on an earlier draft. The authors are also indebted to two anonymous reviewers suggestions of which have allowed an improvement in the quality of the article. The usual disclaimer applies.

\section{References}

[1] Allen, R.G.D., 1959. Mathematical Economics. Palgrave Macmillan. London UK.

[2] Asada, T., Yoshida, H., 2001. Stability, instability and complex behavior in macrodynamic models with policy lag. Discrete Dynamics in Nature and Society 5, 281-295.

[3] Asada, T., Chiarella, C., Flaschel, P., Franke, R., 2010. Monetary Macrodynamics. Routledge, NY, US.

[4] Bi, P., Ruan, S., 2013. Bifurcations in delay differential equations and applications to tumor and immune system interaction models. SIAM Journal of Applied Dynamical Systems 12, $1847-1888$.

[5] Böhm, V., 2006. Multiplier-accelerator models with random perturbations. In: Puu, T., Sushko, I., eds., Business Cycle Dynamics. Models and Tools. Springer-Verlag, Berlin, 113141.

[6] Braddock, R.D. van den Driessche, P., 1983. On a two lag differential delay equation. Journal of the Australian Mathematical Society Series B 24, 292-317.

[7] Cánovas and Ruiz Marín, 2006. Non-autonomous business cycle model. In: Puu, T., Sushko, I., eds., Business Cycle Dynamics. Models and Tools. Springer-Verlag, Berlin, 143-178.

[8] Chiarella, C., Flaschel, P., 2000. High order disequilibrium growth dynamics: theoretical aspects and numerical features. Journal of Economic Dynamics \& Control 24, 935-963.

[9] Chiarella, C., Flaschel, P., Groh, G., Semmler, W., 2000. Disequilibrium, Growth and Labor Market Dynamics: Macro Perspectives. Springer-Verlag, Berlin.

[10] Christiano, L.J., Eichenbaum, M., Evans, C.L., 2005. Nominal rigidities and the dynamic effects of a shock to monetary policy. Journal of Political Economy 113, 1-45.

[11] Estrella, A., Fuhrer, J., 2002. Dynamical Inconsistencies: counterfactual implications of a class of rational expectations models. American Economic Review 92, 1013-1028. 
[12] Ferguson, B.S., Lim, G.C., 2003. Dynamic Economic Models in Discrete Time. Theory and Empirical Applications. Routledge. London, UK.

[13] Gandolfo, G., 2010. Economic Dynamics, 4th edition. Springer, Berlin.

[14] Gori, L., Guerrini, L., Sodini, M., 2015. Hopf bifurcation and stability crossing curves in a cobweb model with heterogeneous producers and time delays. Nonlinear Analysis: Hybrid Systems 18, 117-133.

[15] Gu, K., Niculescu, S.I., Chen, J., 2005. On stability crossing curves for general systems with two delays. Journal of Mathematical Analysis and Applications 311, 231-253.

[16] Györi, I., 1990. Interaction between oscillations and global asymptotic stability in delay differential equations. Differential and Integral Equations 3, 181-200.

[17] He, X., Li, K., 2012. Heterogeneous beliefs and adaptive behaviour in a continuous-time asset price model. Journal of Economic Dynamics \& Control 36, 973-987.

[18] He, X., Li, K., Wei, J., Zheng, M., 2009. Market stability switches in a continuous-time financial market with heterogeneous beliefs. Economic Modelling 26, 1432-1442.

[19] Li, T.Y., Yorke, J.A., 1975. Period three implies chaos. American Mathematical Monthly $82,985-992$.

[20] Mankiw, G., 2001. The inexorable and mysterious tradeoff between inflation and unemployment, Economic Journal 111, 45-61.

[21] Matsumoto, A., Szidarovsky, F. 2016. Learning in monopolies with delayed price information. In : Matsumoto, A., Szidarovsky, F., Asada, T., eds. Essays in Economic Dynamics: Theory, Simulation Analysis, and Methodological Study. Springer, Singapore, 57-79.

[22] Naimzada, A., Pireddu, M., 2014a. Dynamics in a nonlinear Keynesian good market model. Chaos 24, 013142.

[23] Naimzada, A., Pireddu, M., 2014b. Dynamic behavior of product and stock markets with a varying degree of interaction. Economic Modelling 41, 191-197.

[24] Naimzada, A., Pireddu, M., 2015. Real and financial interacting markets: a behavioral macro-model. Chaos, Solitons \& Fractals 77, 111-131.

[25] Piotrowska, M.J., 2007. A remark on the ODE with two discrete delays. Journal of Mathematical Analysis and Applications 329, 664-676.

[26] Ruan, S., 2006. Delay differential equations in single species dynamics. In: Arino, O., Hbid, M., Dads, E.A. (eds.) Delay Differential Equations and Applications. NATO Science Series (II. Mathematics, Physics and Chemistry). Springer, Dordrecht, 477-517.

[27] Shone, R., 2001. An Introduction to Economic Dynamics. Cambridge University Press, NY, US.

[28] Smets, F., Wouters, R., 2003. An estimated dynamic stochastic general equilibrium model for the euro area. Journal of the European Economic Association 1, 1123-1175.

[29] Solow, R., 2004. Introduction: the Tobin approach to monetary economics. Journal of Money, Credit and Banking 36, 657-663. 
[30] Sprott, J.C., 2007. A simple chaotic delay differential equation. Physics Letters A 366, 397402.

[31] Westerhoff, F., 2012. Interactions between the real economy and the stock market: a simple agent-based approach. Discrete Dynamics in Nature and Society 2012, Article ID 504840.

[32] Wolf, A., Swift, J.B., Swinney, H.L., Vastano, J.A., 1985. Determining Lyapunov exponents from a time series. Physica D 16, 285-317.

[33] Wright, E.M., 1955. A non-linear difference-differential equation. Journal für reine und angewandte Mathematik 194, 66-87.

[34] Yorke, J.A., 1970. Asymptotic stability for one dimensional differential-delay equations. Journal of Differential Equations 7, 189-202. 